\title{
Overexpression of RAB34 associates with tumor aggressiveness and immune infiltration in glioma
}

Peng Hou

Wuxi No 2 People's Hospital

Quan Wan

Wuxi No 2 People's Hospital

Qing Wang

Wuxi No 2 People's Hospital

Xuechao Wu ( $\square$ wuxc89@njmu.edu.cn )

Wuxi No.2 People's Hospital https://orcid.org/0000-0002-3655-0851

Xiaojie Lu

Wuxi No 2 People's Hospital

Primary research

Keywords: RAB34, glioma, DNA methylation, immune infiltration, immune checkpoint, EMT

Posted Date: November 9th, 2021

DOI: https://doi.org/10.21203/rs.3.rs-779217/v2

License: (c) (1) This work is licensed under a Creative Commons Attribution 4.0 International License.

Read Full License 


\section{Abstract \\ Background}

RAB34 is aberrantly expressed in various cancers and exhibits oncogenic properties. However, its function in glioma remains largely unclear. Herein, we investigated the clinical value and biological functions of RAB34 in glioma.

\section{Methods}

In this study, we collected 697 RNA-seq data from The Cancer Genome Atlas (TCGA) dataset and 325 RNA-seq data from Chinese Glioma Genome Atlas (CGGA) dataset. CCK-8 and EdU assays were employed to assess cell proliferation ability. The transwell assay was utilized to explore cell migration and invasion capacities. Western blot coupled with qRT-PCR were employed to determine the protein, as well as RNA contents. The statistical analyses along with the graphical work were mainly implemented in the R software.

\section{Results}

RAB34 expression was positively related to the glioma tumor grade, and predicted poor outcomes for glioma patients. RAB34 expression was significantly upregulated in classical and mesenchymal subtypes, and IDH wild-type gliomas. Additionally, RAB34 expression was regulated by promoter DNA methylation. Moreover, RAB34 expression was remarkably correlated with inflammatory activities, immune infiltration, and immune checkpoints in glioma. In vitro experiments demonstrated that inhibition of RAB34 restrained the growth, migration, as well as invasion of glioma cells, and reversed the epithelialto-mesenchymal transition (EMT) process.

\section{Conclusion}

Our findings established RAB34 as a novel progression-related biomarker and a possible immunotherapy target for glioma.

\section{Introduction}

Glioma is the most frequent primary brain tumor, exhibiting high morbidity, a low rate of healing, and a high relapse rate[1,2]. On the basis of histology and pathology, glioma is classified into four grades according to the World Health Organization (WHO)[3]. Glioblastoma multiforme (GBM, WHO grade IV) is the most aggressive and malignant subtype of glioma, whose median survival time is only 12-15 months. In spite of the advancements in surgery, as well as adjuvant therapy, the overall survival (OS) for individuals with glioma has changed slightly in the recent decades[4]. Conventional treatments for glioma 
have not been sufficient to prevent tumor recurrence and progression. Therefore, it is urgent to develop new and more targeted therapies against glioma.

Cancers occur in intricate tissue environments, upon which they depend for sustained growth, infiltration, and metastasis[5]. There is increasing evidence that the tumor microenvironment (TME) plays an indispensable role in supporting the occurrence and progress of glioma[6]. The TME is composed of stromal cells, soluble factors, infiltrating immune cells, extracellular matrices, and other components. Research documented that stromal cells play an imperative role in tumor growth, progression of disease, as well as drug resistance[7]. Additionally, stromal cells in the GBM environments could trigger human GBM cell growth by activating extracellular signaling. Moreover, infiltration of immune cells into tumors may contribute to the establishment of an immunosuppressive TME, which promotes tumor development, metastasis, and resistance to cancer therapies[8-10]. As an important component of TME, tumor-associated macrophages (TAMs) have been shown to create a favorable stroma for growth of tumors and numerous tumor-promoting activities, including angiogenesis, cell infiltration, and repression of adaptive anti-tumor immunity[11]. Increased infiltration of TAMs usually leads to tumor progression and poor prognosis for patients with glioma[12]. In recent years, targeted immunotherapy has received a great deal of attention. However, currently available immunotherapies for glioma have shown only a moderate clinical efficacy. Thus, it is of great clinical value to develop better immunotherapeutic strategies.

Rab small GTPases, which belong to the large Ras protein superfamily, are indispensable modulators of intracellular transport, as well as membrane trafficking in eukaryotic cells. The GTPases of the Rab family can modulate the specificity of the vesicular transport stages in cells. Along with their cross-talk proteins, Rab proteins would possibly constitute the intersection points between vesicular transport and metabolic signaling cascades, including growth factor signaling, glucose and lipid metabolism, as well as autophagy[13]. Recently, increasing studies have reported that Rab proteins have an indispensable role in the onset and progress of tumors[14, 15]. Elevated expression of RAB23 enhanced cell migration along with infiltration of diffuse-type gastric cancer[16]. RAB25 expression was significantly upregulated in ovarian and breast cancers, and accelerated the malignant progression of cancers by mediating AKT signaling and integrin recycling[17]. RAB7 was linked to poor outcomes of gastric cancer, as well as induced cancer cell proliferation, invasion and migration by promoting the phosphorylation of PI3K and AKT[18]. In addition, accumulating evidence indicated that Rab proteins have core roles in immune response, differentiation of immune cells and the modulation of biological processes[19, 20]. These findings imply that Rab proteins may serve as therapeutic targets for cancer.

RAB34, a Rab protein family member, is involved in repositioning of lysosomes, activation of micropinocytosis, and protein transport[21, 22]. Recent investigations have revealed that RAB34 participated in tumor oncogenesis and progression of multiple cancers. For example, RAB34 regulated cell adhesion, migration and invasion by mediating endocytosis and recycling of integrin $\beta 3$ in breast cancer[23]. Overexpression of RAB34 may lead to poor prognosis and tumor progression for liver cancer patients[24]. Moreover, RAB34 promoted cell growth, migration along with tumorigenesis in non-small cell 
lung cancer[25]. However, the underlying function, as well as the mechanism of RAB34 in the progression of glioma remain unclear.

Herein, we assessed the expression and immune features of RAB34 in glioma at the molecular, as well as clinical levels via a large-scale analysis, and further investigated the biological role of RAB34 via in vitro experiments. We established that RAB34 was remarkably upregulated in malignant glioma, and was related to immune infiltration and tumor progression. A profound comprehension of the expression and feature of RAB34 would provide a molecular basis for the future immunotherapy of glioma.

\section{Materials And Methods}

\section{Data collection and human tissue samples}

The TCGA cohort was abstracted from the University of California, Santa Cruz, Xena data resource (https://xenabrowser.net/), with a collection of 697 RNA-seq and related phenotypic data. An additional dataset including 325 RNA-seq data was obtained from the CGGA (Chinese Glioma Genome Atlas) data resource (http://www.cgga.org.cn/).

A total of 49 glioma tissues and 15 normal brain tissues were acquired from the Department of Neurosurgery, The Affiliated Wuxi No.2 People's Hospital of Nanjing Medical university, China. All samples had a confirmed pathological diagnosis as per the 2007 WHO classification by neuropathologists. Nonmalignant brain tissue samples were acquired from 15 adult patients who were being treated with internal decompression surgery after experiencing a severe traumatic brain damage.

\section{Cell culture}

U251, U87, A172, T98 glioma cells were obtained from the American Type Culture Collection (ATCC, USA). HA1800 normal human astrocyte cells were supplied by Boster Biological Technology, Ltd. (Wuhan, China). Cells were cultured in the DMEM medium (Invitrogen, Carlsbad, CA, USA) enriched with 10\% FBS (Gibco, USA) along with $1 \%$ streptomycin-penicillin. All the cells were grown under $5 \% \mathrm{CO}_{2}$ and $37^{\circ} \mathrm{C}$ conditions

\section{RNA isolation and quantitative real-time PCR (qRT-PCR)}

The Trizol reagent (Invitrogen, USA) was employed to isolate total tissue and cellular RNA as described by the manufacturer. The quality along with the quantity of the RNAs were checked on the NanoDrop ND2000 spectrophotometer (NanoDrop Technologies, Houston, TX, USA). After that, cDNA was generated with the PrimeScript ${ }^{\text {TM }}$ RT Master Mix (TaKaRa Biotechnology, Dalian, China), Subsequently, qPCR was done with the QuantiFast SYBR Green PCR Kit (Qiagen, Germany).

\section{Western blot assay}


The RIPA lysis buffer enriched with protease inhibitors (Beyotime Institute of Biotechnology) was employed to isolate total proteins from the cells and tissues, and span at $14000 \mathrm{~g}$ for $15 \mathrm{~min}$ at $4^{\circ} \mathrm{C}$. Afterwards, the BCA protein assay kit (Beyotime Institute of Biotechnology, Jiangsu, China) was employed to quantify the proteins. Thereafter, $40 \mu \mathrm{g}$ of the proteins were fractionated on $12 \%$ SDS-PAGE gels. Then, the fractionated proteins were blotted onto $0.45 \mu \mathrm{m}$ PVDF membranes (Thermo, USA), and $5 \%$ nonfat powdered milk in TBST employed to block the membranes for one hour. After that, the membranes were inoculated overnight with a primary antibody at $4^{\circ} \mathrm{C}$. Thereafter, the membranes were rinsed thrice in TBST dried and incubated for two hours with the respective HRP-labelled secondary antibody (1:5000). Visualization was performed by ECL (Absin, Shanghai, China) and the band intensity was detected by densitometry.

\section{Cell transfection}

Small interfering RNA (siRNA) targeting RAB34 was chemically synthesized by GenePharma (Suzhou, China). Universal sequences were used as negative controls. Lipofectamine 3000 (Invitrogen, Carlsbad, USA) was used to transfect siRNAs into U251 and U87cells. All of the above procedures were performed as documented in the manual provided by the manufacturer.

\section{CCK-8 assay}

The Cell Count Kit-8 was employed to explore cell proliferation as described by the manufacturer. After two days of transfection, 5000 cells/well were inoculated in 96 -well plates for 24,48 , and 72 hours. Next, $10 \mu \mathrm{l} /$ well CCK-8 solution was introduced, followed by incubation for two hours. The OD value of each sample was read on a Spectrophotometer at $450 \mathrm{~nm}$. The experiment was repeated thrice.

\section{EdU assay}

The EdU assay was conducted using the 5-Ethynyl-2'-deoxyuridine kit (C10310; Ribobio) according to the instruction manual. Firstly, cells at a density of $2.5^{\star} 10^{\wedge} 5 /$ well were cultured in 96 -well plates for $36 \mathrm{~h}$. Subsequently, after incubating cells with $100 \mu \mathrm{l} 50 \mu \mathrm{M}$ EdU for two hours, cell fixative and $0.2 \%$ TritonX100 were applied to fix cells for 30 minutes and permeabilize cells for 5 minutes at RT (room temperature) respectively. The cells then were inoculated with Apollo reaction mixture to mark the cells for 30 minutes. Ultimately, the nucleus was stained using Hoechst 33342 and visualized under a fluorescent microscope (Olympus).

\section{Transwell assay}

Cell migration along with invasion abilities were tested by transwell assay. For migration assay, cells were re-suspended in $200 \mu \mathrm{L}$ serum-free medium and inoculated into the upper chamber. $600 \mu \mathrm{L}$ DMEM with $10 \%$ FBS was then introduced into the lower chamber and the cells were incubated for $12 \mathrm{~h}$. For invasion assay, Matrigel solution (BD, USA) was pre-coated in the upper chamber. Cells on the bottom surface were fixed with methanol and stained using $0.1 \%$ crystal violet. 5 random fields per chamber were chosen冈and observed under an inverted microscope (Olympus). All experiments were replicated thrice. 


\section{Statistical analysis}

In the present study, the statistical analyses were mainly implemented in the R language. The R packages consisting of ggplot2, circlize, pheatmap, corrgram, corrplot and pROC were employed to generate figures. For in vitro experiments, the statistical analysis was implemented in the GraphPad Prism V.7.0. Student's t test or one-way analysis of variance was applied for analyzing the data. $\mathrm{P}<0.05$ defined statistical significance.

\section{Results}

\section{The expression of RAB34 is upregulated in glioma}

RAB34 expression data was abstracted from the TCGA and CGGA data resources. We found that RAB34 expression was remarkably higher in GBM (WHO IV) in contrast with that in WHO grade II, as well as grade III gliomas (Fig. 1a, b). Besides, the increased expression, RAB34 was positively correlated with advanced WHO grade of glioma. To further confirm these results, we examined RAB34 expression level in glioma samples, as well as cell lines via qRT-PCR. The data illustrated that RAB34 expression was dramatically increased in glioma samples than that in normal brain tissues (Fig. 1c). Furthermore, grade IV GBM samples showed the highest RAB34 expression, which is consistent with above results (Fig. 1d). Moreover, RAB34 expression was upregulated in U251, U87, A172, H4 and SHG44 glioma cells than that in HA1800 (Fig. 1e). These results suggested that the abnormal expression of RAB34 might be involved in glioma progression.

\section{RAB34 expression is enriched in IDH wild-type glioma}

Next, we examined RAB34 distribution in four TCGA-defined molecular subtypes. The results revealed that RAB34 was apparently upregulated in classical and mesenchymal subtypes than the other two subtypes in TCGA and CGGA datasets (Fig. 2a, b). It is acknowledged that IDH mutation status is a clinically relevant molecular marker in glioma progression. Therefore, we further explored the relationship of RAB34 expression with IDH mutation status. We established that IDH wild-type glioma exhibited a higher content of RAB34 in contrast with IDH mutant glioma in TCGA and CGGA datasets (Fig. 2c, d). Subsequently, receiver operating characteristic (ROC) analysis was employed to explore the diagnostic significance of RAB34 as a signature for diagnosing IDH status in glioma. Results showed that areas under curve (AUCs) were $95.1 \%$ and $97.6 \%$ in TCGA and CGGA cohorts, respectively (Fig. 2e, f). These results suggested that RAB34 was selectively distributed and could serve as a signature for IDH wild-type glioma.

\section{RAB34 expression is negatively correlated with promoter DNA methylation}

To further investigate the possible mechanism of high RAB34 levels in glioma, we integrated TCGA glioma data sets and identified 594 patients with RAB34 expression along with DNA methylation data. In particular, six CpG sites (cg19982230, cg08032476, cg22803868, cg03452174, cg08839210, and cg21237418) were located in the promoter region of RAB34. Interestingly, we found that the DNA 
methylation beta values at all these six sites were markedly higher in LGG (lower grade glioma) than that in GBM (Supplementary Figure S1). Additionally, the promoter DNA methylation beta values were higher in the low RAB34 expression group compared with that in the high RAB34 expression group (Fig. 3a). Moreover, all the beta values at promoter region were negatively related to RAB34 mRNA expression (Fig. 3b). To further validate these results, we treated U251 and U87 cells with DAC (decitabine), which is a DNA methyltransferase inhibitor. Western blot results showed that RAB34 expression was increased in response to DAC in a dose of $5 \mu \mathrm{M}$ or $10 \mu \mathrm{M}$ compared with untreated cells (Fig. 3c). Meanwhile, we found that patients who had low methylation levels exhibited a dramatically worse OS in contrast with those who had high methylation levels (Fig. 3d). In summary, these data implied that RAB34 expression was possibly modulated by promoter DNA methylation, and this epigenetic modification could also serve as a promising prognostic signature for patients with glioma.

\section{RAB34 expression is related to immune functions and inflammatory activities in glioma}

To identify the biological effect of RAB34 in glioma, GO analysis was conducted. First, RAB34-linked genes in the cohorts were explored with Pearson correlation, with a cut-point of $|R|>0.5$ along with $P$ $<0.01$. Overall, 2220 and 743 positive genes, as well as 1629 and 289 negative genes were detected as remarkably linked to expression of RAB34 in TCGA and CGGA cohorts, respectively. Then, we investigated the biological roles of these genes with the DAVID data resource. The results demonstrated that the genes positively correlated with RAB34 in TCGA dataset were enriched in extracellular matrix organization, immune response, angiogenesis, and inflammatory response (Fig. 4a). Meanwhile, the positive genes in CGGA dataset were enriched in positive modulation of I-kappaB kinase/NF-kappaB signaling, cell-cell adhesion, extracellular matrix organization, and response to hypoxia (Fig. 4b).

To further examine if RAB34 is associated with inflammatory response, seven clusters (including 104 genes) that were defined as metagenes were selected to represent diverse kinds of inflammation and immune response. RAB34 expression was positively linked to most of the clusters except for IgG in TCGA and CGGA datasets (Fig. 4c, d). To verify these data, Gene Sets Variation Analysis (GSVA) was employed to convert these metagenes' expression levels into enrichment scores, and then corrgrams were generated on the basis of the Pearson correlation values between RAB34 and seven metagenes. As illustrated in Figure $4 \mathrm{e}$ and $\mathrm{f}$, RAB34 expression was positively related to HCK, LCK, MHC-I, MCH-II, STAT1, and interferon but negatively related to IgG, a marker for $B$ cells. Taken together, these results indicated that RAB34 played an important role in the inflammatory response in glioma.

\section{RAB34 expression is correlated with stromal cells and immune cells in glioma}

Glioma tissues include not only glioma cells, but also non-tumor cells associated with gliomas, e.g., stromal cells along with immune cells. These non-tumor cells dilute the purity of glioma cells and serve a vital role in glioma biology[26]. Previous researches reported that tumor purity was closely related to major clinical, as well as molecular characteristics, and low-purity cases were more likely to be diagnosed as malignant tumors and independently associated with shorter survival time[27]. Considering the GO analysis data, we next investigated whether RAB34 expression was linked to the infiltrated cells in glioma 
using ESTIMATE algorithm method described by Yoshihara[28]. The results demonstrated that RAB34 was remarkably related to the stromal score, immune score, and ESTIMATE score in both TCGA and CGGA datasets (Fig. 5a, b). We further employed the CIBERSORT algorithm[29] to investigate the relative proportions of 22 subpopulations of infiltrating immune cells in glioma tissues from TCGA cohort. A comparative summary of the percentages of immune cells in different RAB34 expression groups was briefly depicted in Figure 5c. Notably, we identified that the percentages of $M 0$ and $M 2$ macrophages, and T cells CD4 memory resting were dramatically elevated in the high RAB34 expression group, while monocytes and plasma cells were remarkably enriched in the low RAB34 expression group. Next, we analyzed the relationship of RAB34 expression with 22 non-malignant cell types. The results showed that 10 types were positively related to RAB34 expression, whereas 6 types were negatively related (Fig. 5d). These findings strongly indicated that RAB34 played a crucial role in the infiltration of stromal cells and immune cells in the glioma microenvironment.

\section{RAB34 expression is related to immune checkpoints in glioma}

Immune checkpoint inhibitors designed to restore tumor-induced immunosuppression have become effective anticancer therapies[30]. Therefore, we explored the relationship of RAB34 with immune checkpoints that have been investigated in clinical trials or clinical situations, including PD-1, PD-L1, CTLA4, PD-L2, TIM-3, and B7-H3. Intriguingly, RAB34 exhibited closely positive relationship with these immune checkpoints in all grade gliomas in TCGA and CGGA cohorts (Fig. 6a, b). Similar findings were observed in GBM samples (Fig. 6c, d). These results suggested the possible modulatory influences of RAB34 on these checkpoint members.

\section{Suppression of RAB34 inhibits EMT and the biological function of glioma cells}

Firstly, we evaluated the relationship between RAB34 and EMT. Consistently, we found that RAB34 expression was dramatically linked to common EMT biomarkers based on TCGA and CGGA datasets (Supplementary Figure S2A and B). Furthermore, we conducted western blot assays to assess the influence of RAB34 on the EMT process in glioma cells. As shown in Figure 7a, small interfering RNA siRAB34 and negative control (si-NC) were inserted into U251 and U87 cells via transfection, respectively. si-RAB34 significantly reduced the expression of $\mathrm{N}$-cadherin along with that of Vimentin, but elevated the expression of Claudin-1 in U251 and U87 cell lines. In addition, CCK-8 assays showed that the viability of glioma cells was remarkably reduced after knockdown of RAB34 (Fig. 7b). EdU assays also illustrated that the number of cells excited by red light in the si-RAB34 group were much less than that in the si-NC group (Fig. 7c). In the transwell assays, we found that the reduction of RAB34 dramatically repressed the migration and invasion abilities of U251 and U87 cells (Fig. 7d). These results revealed that RAB34 may contribute to the EMT progression of glioma and promote cell proliferation, migration and invasion.

\section{Overexpression of RAB34 indicates worse prognosis for glioma patients Considering that RAB34} expression is abnormally expressed in glioma and correlates with histological grade, as well as molecular subtype, we speculated that RAB34 may be an indispensable prognostic signature for glioma patients. Thus, Kaplan-Meier curves were generated to explore its prognostic value. As illustrated in Figure $8 a$ and 
b, patients with higher RAB34 expression had a dramatically shorter OS in contrast with patients with lower RAB34 expression in TCGA and CGGA cohorts. Simultaneously, we performed univariate and multivariate analyses to explore whether RAB34 could be an independent predictive factor for glioma patients. Univariate analysis indicated that RAB34 expression was remarkably correlated to OS (Fig. 8c, d). In multivariate analysis, RAB34 expression was still a remarkable prognostic factor after correcting for other clinical factors, such as age, gender and glioma grade (Fig. 8e, f). In summary, RAB34 was an independent predictive factor for patients with glioma.

\section{Discussion}

Glioma is the most common primary intracranial malignant tumor with a generally poor prognosis. Presenting extremely high mortality rates, GBM is the most aggressive subtype of brain tumor. Even with intensive therapies, consisting of neurosurgical resection, targeted treatment, adjuvant chemotherapy, as well as radiotherapy, most individuals with GBM experience relapse given the highly aggressive nature of this malignancy[31]. Immunotherapy, as one of the most potential strategies of activating anti-tumor immune response, has shown remarkable success for treating numerous cancers including glioma[3234]. However, low response rate and treatment resistance originating from the precise interaction of cytokines with immune cells severely limited the widespread application of immunotherapy[35]. At present, one promising treatment approach is to design immunotherapy against glioma through targeting the highly expressed proteins that have an indispensable role in immune repression. Therefore, it is urgently needed to evaluate novel targets of immunotherapy.

RAB34, a type of RAB proteins, has been documented to be expressed abnormally and play critical roles in diverse kinds of cancer. However, its function remained unclear in glioma. Herein, we comprehensively analyzed the oncogenic role of RAB34 in the malignant progress of glioma by conducting a large-scale analysis and in vitro experiments. The data demonstrated that the expression of RAB34 was not only aberrantly overexpressed in glioma, but also dramatically correlated with WHO grade. IDH wild-type glioma is recognized generally to have a worse OS in contrast with IDH mutant glioma. Our results illustrated that RAB34 was highly enriched in IDH wild-type glioma. In addition, high RAB34 expression predicted a worse prognosis, which is consistent with its enrichment in IDH wild-type glioma. Furthermore, our research certified that RAB34 was an independent predictive marker for individuals with glioma. Accumulating studies have shown that DNA methylation, the primary epigenetic modification, participates in the initiation and progression of different tumors. Moreover, aberrantly methylated promoters have been identified as prospective biomarkers for the diagnosis and prognosis of glioma[36, 37]. Therefore, we explored the potential mechanism of RAB34 overexpression, and found that RAB34 expression was significantly negatively related to the promoter methylation levels. Additionally, the promoter methylation levels of RAB34 in GBM was significantly lower than that in LGG. Furthermore, we verified that the expression of RAB34 was significantly increased after treatment with DAC in glioma cell lines. Our results indicated that hypomethylation of RAB34 promoter region may be a key contributor to the increased RAB34 expression in glioma. 
Immunotherapy is becoming the most potential therapy for malignant tumors. Nevertheless, the TME, composed of extracellular matrix, stromal cells, vasculature, and inflammatory cells, is often involved in supporting the progress of glioma. Therefore, it is of great importance to identify novel molecular biomarkers, as well as targets that make an important impact in the TME. Herein, we established that RAB34 played an indispensable role in inflammatory response, immune response and angiogenesis, as well as remarkably positively correlated with infiltrated stromal cells along with immune cells. The immune escape mechanism of tumorigenesis has been demonstrated by numerous studies, and a number of immunosuppressive agents have been applied clinically in recent years. Immune checkpoint inhibitors in particular have demonstrated remarkable success in treating many tumors[38, 39]. For instance, PD-1 and PD-L1 inhibitors showed significant improvement in melanoma, non-small cell lung cancer $\square k i d n e y$ cancer and other solid malignancies[40, 41]. Monoclonal antibodies directed against CTLA4, such as ipilimumab, presented significant clinical benefit for individuals with metastatic melanoma[42]. However, due to the side effects of autoimmunity, it is difficult for immunotherapy to be widely used. Accumulating research is focusing on studying a combination of immune checkpoint blockades, which may provide a potential therapy for glioma[43-45]. Given the remarkable efficacy of immune checkpoint blockade treatment, we demonstrated that RAB34 expression was tightly associated with the checkpoint proteins PD-1, TIM-3, PD-L1, B7-H3, PD-L2, and CTLA4, and demonstrating the promising impact of RAB34 on modulating these immune checkpoints.

Recently, increasing research evidence has illustrated that the crosstalk of EMT-related factors with the TME could facilitate tumor immune escape, indicating EMT could play a key role in tumor immunosuppression and immune evasion[46, 47]. In addition, previous studies have shown that EMT is linked to the activation of various immune checkpoint molecules such as PD-L1[48]. EMT-triggered immune evasion enhances the progress of cancer and might also provide a platform for the discovery of new therapies and biomarkers for predicting response to checkpoint inhibitors. Herein, we found that RAB34 expression was remarkably related to EMT markers and promoted EMT process in glioma. Furthermore, RAB34 promoted the proliferation, migration, and infiltration of glioma cells. These findings indicated that RAB34 may play a pro-cancer role in glioma.

\section{Conclusion}

In conclusion, this study discovered a novel function for RAB34 in enhancing the malignant phenotype and resulting in immune evasion of glioma. Our findings highlighted the potential of RAB34 as a novel immunotherapeutic target for glioma.

\section{Abbreviations}

CGGA, Chinese Glioma Genome Atlas

TCGA, The Cancer Genome Atlas

EMT, epithelial-to-mesenchymal transition

GBM, glioblastoma multiforme 
LGG, lower grade glioma

TME, tumor microenvironment

TAM, tumor-associated macrophage

$\mathrm{ROC}$, receiver operating characteristic

AUC, area under curve

$\mathrm{IDH}$, isolated diastolic hypertension

DAC, decitabine

GO, gene ontology

DAVID, The Database for Annotation, Visualization and Integrated Discovery

GSVA, Gene Sets Variation Analysis

OS, overall survival.

\section{Declarations}

\section{Acknowledgments}

We appreciate the generosity of TCGA and CGGA networks for sharing the great amount of data.

\section{Authors' Contributions}

Peng Hou and Qing Wang designed the study, planned the experiments, and wrote the manuscript. Peng Hou and Quan Wan performed the experiments and analyzed the data. Xuechao Wu and Xiaojie Lu made substantial contributions to the conception and design of the present study and revised the manuscript. All authors critically reviewed the manuscript.

\section{Funding}

This work was supported by Jiangsu Province's Science and Technology Project (SBK2019021295); and National Natural Science Foundation of China (82072791).

\section{Availability of data and materials}

The datasets supporting the conclusions of this article are available in TCGA (https://xenabrowser.net/) and CGGA (http://www.cgga.org.cn/).

\section{Data Availability Statement}

The data that supports the findings of this study are available from the corresponding author upon reasonable request.

\section{Ethics approval and consent to participate}

This study was approved by the Ethics Committee of The Affiliated Wuxi No.2 People's Hospital of Nanjing Medical University, and informed consent was obtained from all the patients. 


\section{Consent for publication}

All authors agreed to publish the article in the journal.

\section{Competing interests}

The authors declare that they have no competing interests.

\section{Author details}

${ }^{1}$ Department of Neurosurgery, The Affiliated Wuxi No.2 People's Hospital of Nanjing Medical University, Wuxi 214002, China

${ }^{2}$ Department of Neurosurgery, The Affiliated Wuxi Clinical College of Nantong University, Wuxi 214002, China

\section{References}

1. Liu J, Qu C, Han C, Chen MM, An LJ, Zou W: Potassium channels and their role in glioma: A mini review. Molecular membrane biology 2019, 35(1):76-85.

2. Zhong QY, Fan EX, Feng GY, Chen QY, Gou XX, Yue GJ, Zhang GH: A gene expression-based study on immune cell subtypes and glioma prognosis. BMC Cancer 2019, 19(1):1116.

3. Wen PY, Huse JT: 2016 World Health Organization Classification of Central Nervous System Tumors. Continuum 2017, 23(6, Neuro-oncology):1531-1547.

4. Brandes $\mathrm{A}$, Soesan M, Fiorentino MV: Medical treatment of high grade malignant gliomas in adults: an overview. Anticancer research 1991, 11(2):719-727.

5. Quail DF, Joyce JA: Microenvironmental regulation of tumor progression and metastasis. Nature medicine 2013, 19(11):1423-1437.

6. Akins EA, Aghi MK, Kumar S: Incorporating Tumor-Associated Macrophages into Engineered Models of Glioma. iScience 2020, 23(12):101770.

7. Ma Q, Long W, Xing C, Chu J, Luo M, Wang HY, Liu Q, Wang RF: Cancer Stem Cells and Immunosuppressive Microenvironment in Glioma. Frontiers in immunology 2018, 9:2924.

8. Huang S, Song Z, Zhang T, He X, Huang K, Zhang Q, Shen J, Pan J: Identification of Immune Cell Infiltration and Immune-Related Genes in the Tumor Microenvironment of Glioblastomas. Frontiers in immunology 2020, 11:585034.

9. Jeanmougin M, Havik AB, Cekaite L, Brandal P, Sveen A, Meling TR, Agesen TH, Scheie D, Heim S, Lothe RA et al: Improved prognostication of glioblastoma beyond molecular subtyping by transcriptional profiling of the tumor microenvironment. Molecular oncology 2020, 14(5):1016-1027.

10. Deng X, Lin D, Zhang X, Shen X, Yang Z, Yang L, Lu X, Yu L, Zhang N, Lin J: Profiles of immunerelated genes and immune cell infiltration in the tumor microenvironment of diffuse lower-grade 
gliomas. Journal of cellular physiology 2020, 235(10):7321-7331.

11. Kennedy BC, Showers CR, Anderson DE, Anderson L, Canoll P, Bruce JN, Anderson RC: Tumorassociated macrophages in glioma: friend or foe? Journal of oncology 2013, 2013:486912.

12. Lu-Emerson C, Snuderl M, Kirkpatrick ND, Goveia J, Davidson C, Huang Y, Riedemann L, Taylor J, Ivy $P$, Duda DG et al: Increase in tumor-associated macrophages after antiangiogenic therapy is associated with poor survival among patients with recurrent glioblastoma. Neuro Oncol 2013, 15(8):1079-1087.

13. Chua CE, Tang BL: Role of Rab GTPases and their interacting proteins in mediating metabolic signalling and regulation. Cellular and molecular life sciences: CMLS 2015, 72(12):2289-2304.

14. Cernochova R, Nekulova M, Holcakova J: [Rab Proteins, Intracellular Transport and Cancer]. Klinicka onkologie: casopis Ceske a Slovenske onkologicke spolecnosti, 29 Suppl 4(Suppl 4):31-39.

15. Goud B, Liu S, Storrie B: Rab proteins as major determinants of the Golgi complex structure. Small GTPases 2018, 9(1-2):66-75.

16. Bin Z, Dedong H, Xiangjie F, Hongwei X, Qinghui Y: The microRNA-367 inhibits the invasion and metastasis of gastric cancer by directly repressing Rab23. Genet Test Mol Biomarkers 2015, 19(2):69-74.

17. Cheng KW, Lahad JP, Kuo WL, Lapuk A, Yamada K, Auersperg N, Liu J, Smith-McCune K, Lu KH, Fishman D et al: The RAB25 small GTPase determines aggressiveness of ovarian and breast cancers. Nature medicine 2004, 10(11):1251-1256.

18. Liu H, Xu J, Yao Q, Zhang Z, Guo Q, Lin J: Rab7 Is Associated with Poor Prognosis of Gastric Cancer and Promotes Proliferation, Invasion, and Migration of Gastric Cancer Cells. Med Sci Monit 2020, 26:e922217.

19. Chen C, Eldein S, Zhou X, Sun Y, Gao J, Sun Y, Liu C, Wang L: Immune function of a Rab-related protein by modulating the JAK-STAT signaling pathway in the silkworm, Bombyx mori. Archives of insect biochemistry and physiology 2018, 97(1).

20. Pei G, Bronietzki M, Gutierrez MG: Immune regulation of Rab proteins expression and intracellular transport. Journal of leukocyte biology 2012, 92(1):41-50.

21. Goldenberg NM, Grinstein S, Silverman M: Golgi-bound Rab34 is a novel member of the secretory pathway. Mol Biol Cell 2007, 18(12):4762-4771.

22. Sun P, Yamamoto $H$, Suetsugu $S$, Miki H, Takenawa T, Endo T: Small GTPase Rah/Rab34 is associated with membrane ruffles and macropinosomes and promotes macropinosome formation. $J$ Biol Chem 2003, 278(6):4063-4071.

23. Sun L, Xu X, Chen Y, Zhou Y, Tan R, Qiu H, Jin L, Zhang W, Fan R, Hong W et al: Rab34 regulates adhesion, migration, and invasion of breast cancer cells. Oncogene 2018, 37(27):3698-3714.

24. Wu J, Lu Y, Qin A, Qiao Z, Jiang X: Overexpression of RAB34 correlates with poor prognosis and tumor progression in hepatocellular carcinoma. Oncology reports 2017, 38(5):2967-2974. 
25. Xi Y, Shen W, Jin C, Wang L, Yu B: PVT1 Promotes the Proliferation and Migration of Non-Small Cell Lung Cancer via Regulating miR-148/RAB34 Signal Axis. Onco Targets Ther 2020, 13:1819-1832.

26. Zhang C, Cheng W, Ren X, Wang Z, Liu X, Li G, Han S, Jiang T, Wu A: Tumor Purity as an Underlying Key Factor in Glioma. Clinical cancer research: an official journal of the American Association for Cancer Research 2017, 23(20):6279-6291.

27. Xiong Z, Xiong Y, Liu H, Li C, Li X: Identification of purity and prognosis-related gene signature by network analysis and survival analysis in brain lower grade glioma. Journal of cellular and molecular medicine 2020.

28. Becht E, Giraldo NA, Lacroix L, Buttard B, Elarouci N, Petitprez F, Selves J, Laurent-Puig P, SautesFridman C, Fridman WH et al: Estimating the population abundance of tissue-infiltrating immune and stromal cell populations using gene expression. Genome Bio/ 2016, 17(1):218.

29. Newman AM, Liu CL, Green MR, Gentles AJ, Feng W, Xu Y, Hoang CD, Diehn M, Alizadeh AA: Robust enumeration of cell subsets from tissue expression profiles. Nat Methods 2015, 12(5):453-457.

30. Ahn BJ, Pollack IF, Okada H: Immune-checkpoint blockade and active immunotherapy for glioma. Cancers 2013, 5(4):1379-1412.

31. Lefranc F, Rynkowski M, DeWitte 0 , Kiss R: Present and potential future adjuvant issues in high-grade astrocytic glioma treatment. Advances and technical standards in neurosurgery 2009, 34:3-35.

32. Rathi N, McFarland TR, Nussenzveig R, Agarwal N, Swami U: Evolving Role of Immunotherapy in Metastatic Castration Refractory Prostate Cancer. Drugs 2020.

33. Dai X, Guo Y, Hu Y, Bao X, Zhu X, Fu Q, Zhang H, Tong Z, Liu L, Zheng Y et al: Immunotherapy for targeting cancer stem cells in hepatocellular carcinoma. Theranostics 2021, 11(7):3489-3501.

34. Dunn GP, Cloughesy TF, Maus MV, Prins RM, Reardon DA, Sonabend AM: Emerging immunotherapies for malignant glioma: from immunogenomics to cell therapy. Neuro Oncol 2020, 22(10):1425-1438.

35. Pitt JM, Vetizou M, Daillere R, Roberti MP, Yamazaki T, Routy B, Lepage P, Boneca IG, Chamaillard M, Kroemer G et al: Resistance Mechanisms to Immune-Checkpoint Blockade in Cancer: Tumor-Intrinsic and -Extrinsic Factors. Immunity 2016, 44(6):1255-1269.

36. Dor Y, Cedar H: Principles of DNA methylation and their implications for biology and medicine. Lancet 2018, 392(10149):777-786.

37. Dong X, Deng Q, Nie X, Zhang M, Jia W, Chen C, Xu C, Xu R: Downregulation of HTATIP2 expression is associated with promoter methylation and poor prognosis in glioma. Exp Mol Pathol 2015, 98(2):192-199.

38. Himmel ME, Saibil SD, Saltman AP: Immune checkpoint inhibitors in cancer immunotherapy. CMAJ: Canadian Medical Association journal = journal de l'Association medicale canadienne 2020, 192(24):E651.

39. Huang J, Liu F, Liu Z, Tang H, Wu H, Gong Q, Chen J: Immune Checkpoint in Glioblastoma: Promising and Challenging. Front Pharmacol 2017, 8:242. 
40. Carril-Ajuria L, Lora D, Carretero-Gonzalez A, Martin-Soberon M, Rioja-Viera P, Castellano D, de Velasco G: Systemic Analysis and Review of Nivolumab-ipilimumab Combination as a Rescue Strategy for Renal Cell Carcinoma After Treatment With Anti-PD-1/PD-L1 Therapy. Clinical genitourinary cancer 2020.

41. Zhang SY, Wang Y: [Progress of PD-1/PD-L1 inhibitors in the treatment of small-cell lung cancer]. Zhonghua zhong liu za zhi [Chinese journal of oncology] 2021, 43(1):98-103.

42. Ott PA, Hodi FS, Robert C: CTLA-4 and PD-1/PD-L1 blockade: new immunotherapeutic modalities with durable clinical benefit in melanoma patients. Clinical cancer research: an official journal of the American Association for Cancer Research 2013, 19(19):5300-5309.

43. Strauss J, Madan RA, Gulley JL: Considerations for the combination of anticancer vaccines and immune checkpoint inhibitors. Expert opinion on biological therapy 2016, 16(7):895-901.

44. Rice AE, Latchman YE, Balint JP, Lee JH, Gabitzsch ES, Jones FR: An HPV-E6/E7 immunotherapy plus PD-1 checkpoint inhibition results in tumor regression and reduction in PD-L1 expression. Cancer gene therapy 2015, 22(9):454-462.

45. Soares KC, Rucki AA, Wu AA, Olino K, Xiao Q, Chai Y, Wamwea A, Bigelow E, Lutz E, Liu L et al: PD1/PD-L1 blockade together with vaccine therapy facilitates effector T-cell infiltration into pancreatic tumors. Journal of immunotherapy 2015, 38(1):1-11.

46. Hass R, von der Ohe J, Ungefroren H: The Intimate Relationship Among EMT, MET and TME: A $\mathrm{T}$ (ransdifferentiation) E(nhancing) M(ix) to Be Exploited for Therapeutic Purposes. Cancers 2020, 12(12).

47. Terry S, Savagner P, Ortiz-Cuaran S, Mahjoubi L, Saintigny P, Thiery JP, Chouaib S: New insights into the role of EMT in tumor immune escape. Molecular oncology 2017, 11(7):824-846.

48. Jiang Y, Zhan H: Communication between EMT and PD-L1 signaling: New insights into tumor immune evasion. Cancer letters 2020, 468:72-81.

\section{Figures}



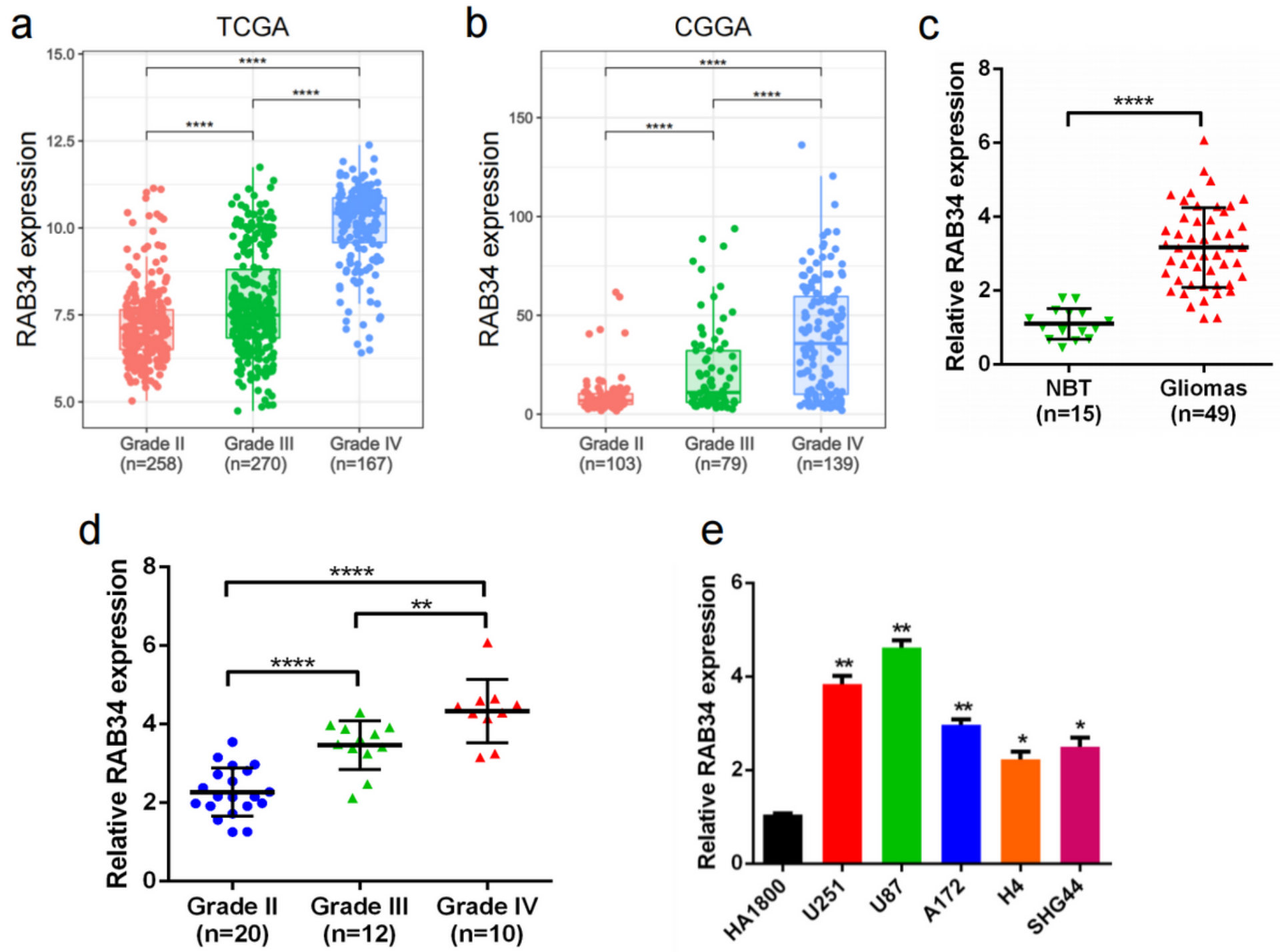

Figure 1

RAB34 expression is significantly increased in glioma. a, b Comparison of RAB34 expression in TCGA and CGGA datasets with different WHO grades. c Validation of RAB34 mRNA expression in 49 glioma samples compared to that in 15 normal brain tissues (NBT) via qRT-PCR. d RAB34 mRNA expression in glioma of WHO grade II-IV was determined based on our patient samples. e The mRNA expression levels of RAB34 in different glioma cell lines and normal glial cell were determined by qRT-PCR. ${ }^{*} \mathrm{P}<0.05$; $* \star \mathrm{P}<$ $0.01 ; * \star \star \star ~ P<0.0001$. 

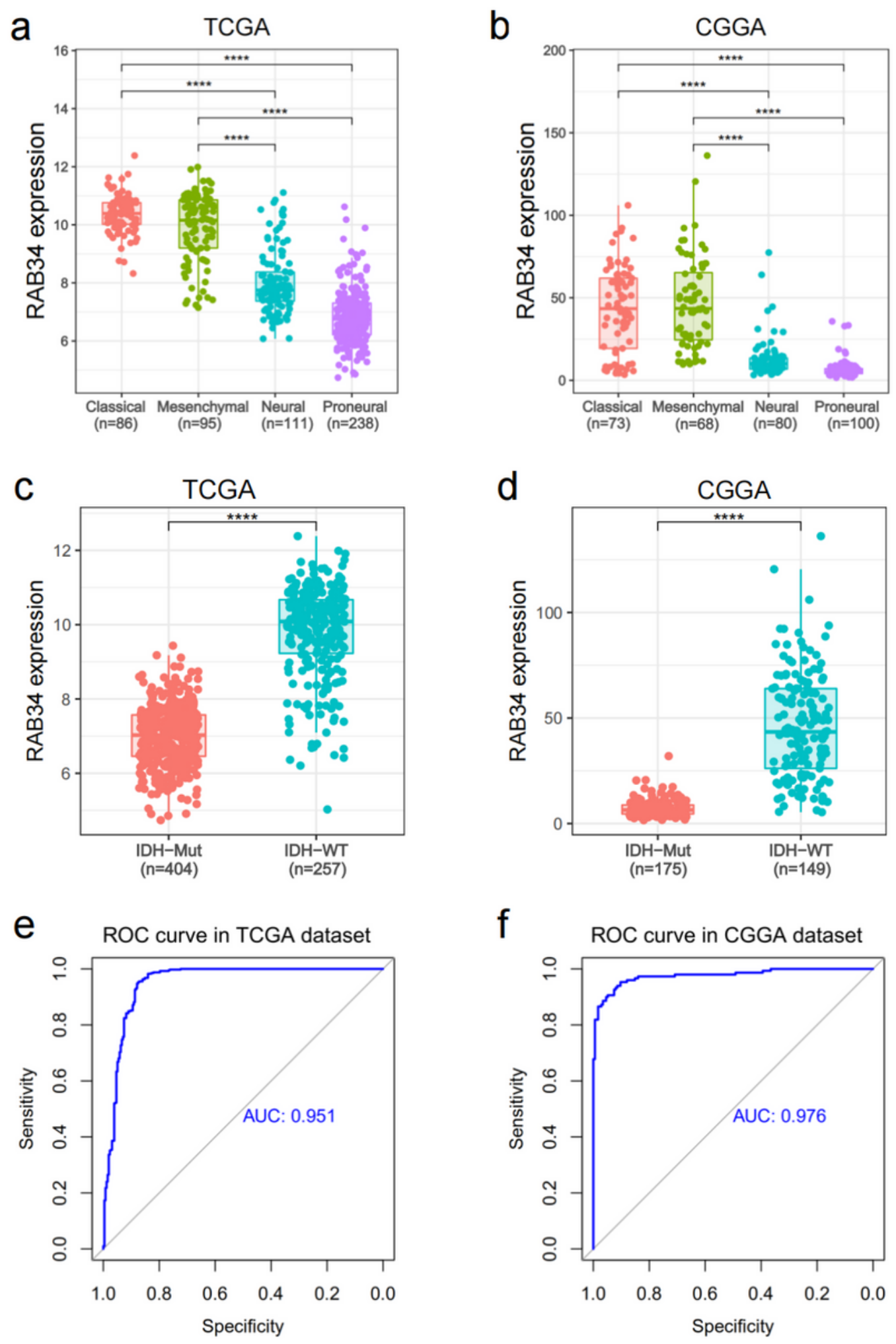

\section{Figure 2}

RAB34 expression is selectively distributed in different subtypes of glioma. a, b RAB34 is overexpressed in classical and mesenchymal subtypes in TCGA and CGGA datasets. c, d RAB34 expression is significantly higher in IDH wild-type glioma than that in IDH mutant glioma in TCGA and CGGA datasets. e, $f$ ROC curve analysis showed that RAB34 could serve as a signature for IDH wild-type glioma in TCGA and CGGA datasets. $* \star \star \star ~ P<0.0001$. 
a

RAB34 审 high 审 low
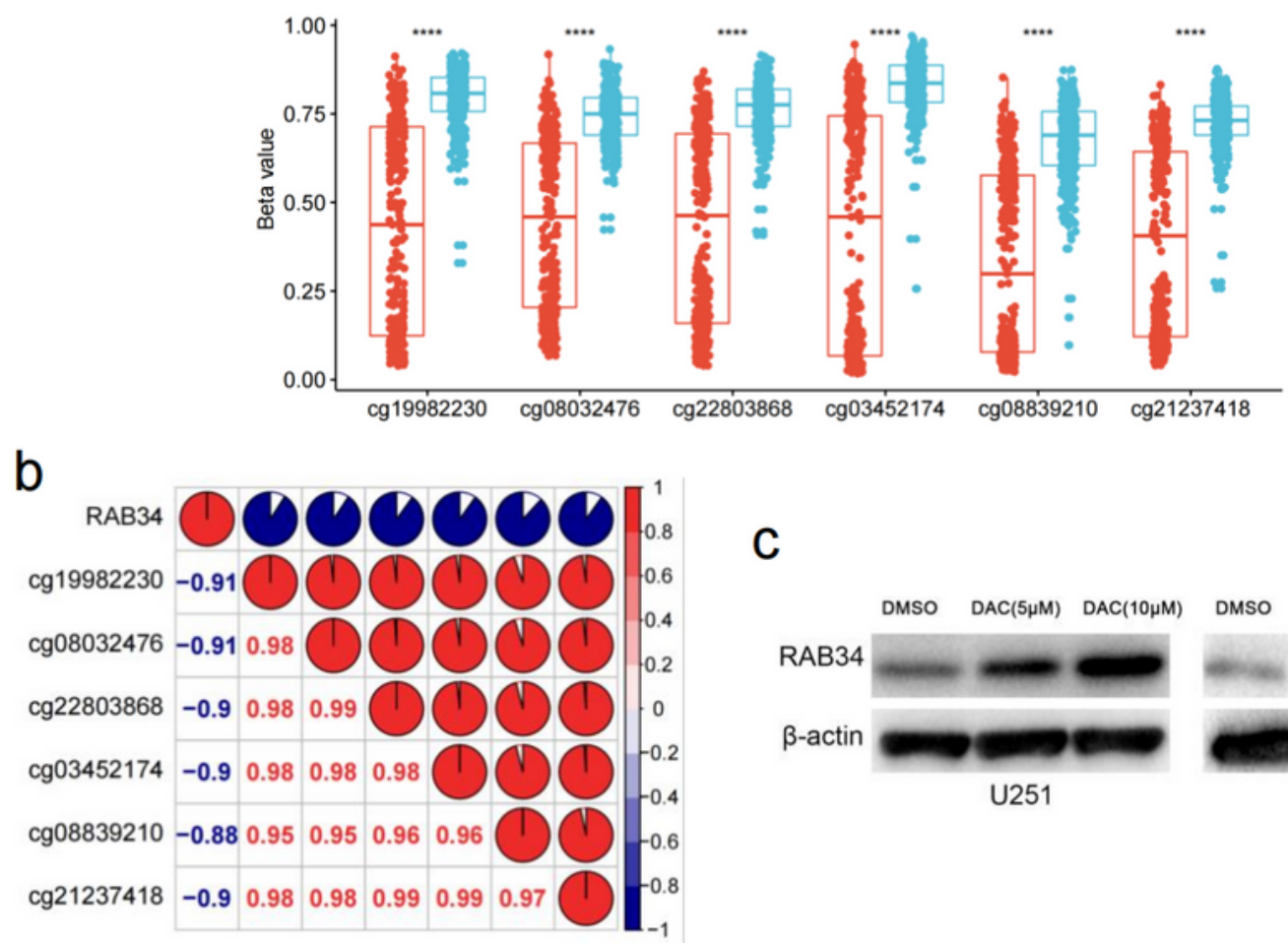

C

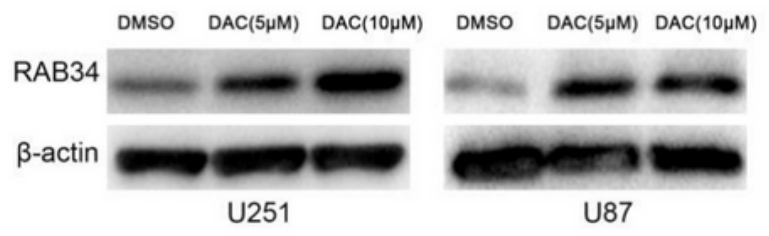

d
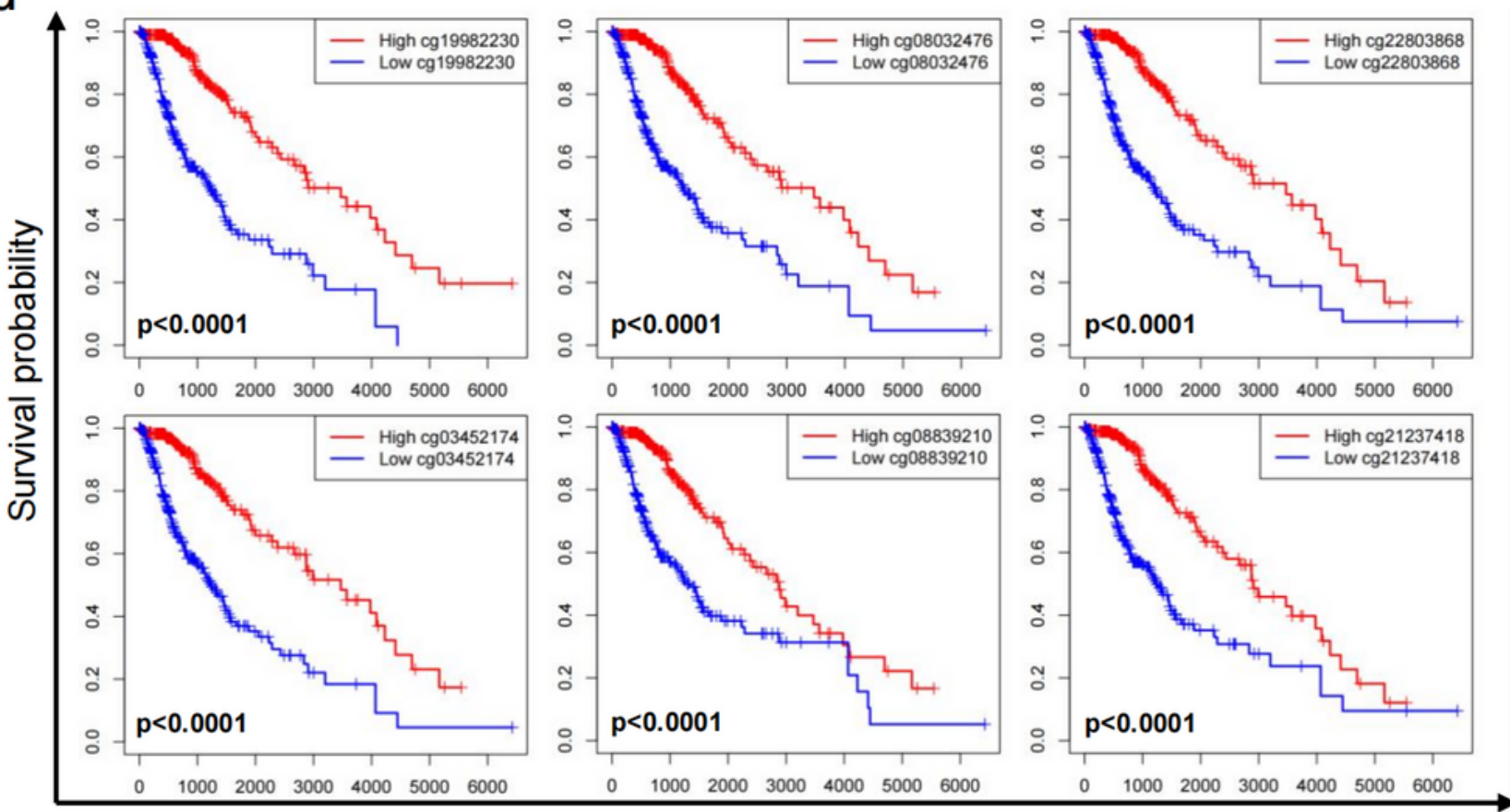

Time (days)

\section{Figure 3}

Promoter DNA methylation is correlated with RAB34 expression and glioma patients' prognosis. a Comparison of DNA methylation beta values at six CpG sites (cg19982230, cg08032476, cg22803868, cg03452174, cg08839210, and cg21237418) between low and high RAB34 expression groups. b Correlation between RAB34 expression and the promoter DNA methylation beta values in TCGA dataset. C Western blot results showed that RAB34 expression was increased in response to DAC (decitabine) in a 
dose of $5 \mu \mathrm{M}$ or $10 \mu \mathrm{M}$ in U251 and U87 cell lines. $d$ The promoter DNA methylation levels of RAB34 was associated with overall survival time for glioma patients. $\star \star \star \star ~ P<0.0001$.

a

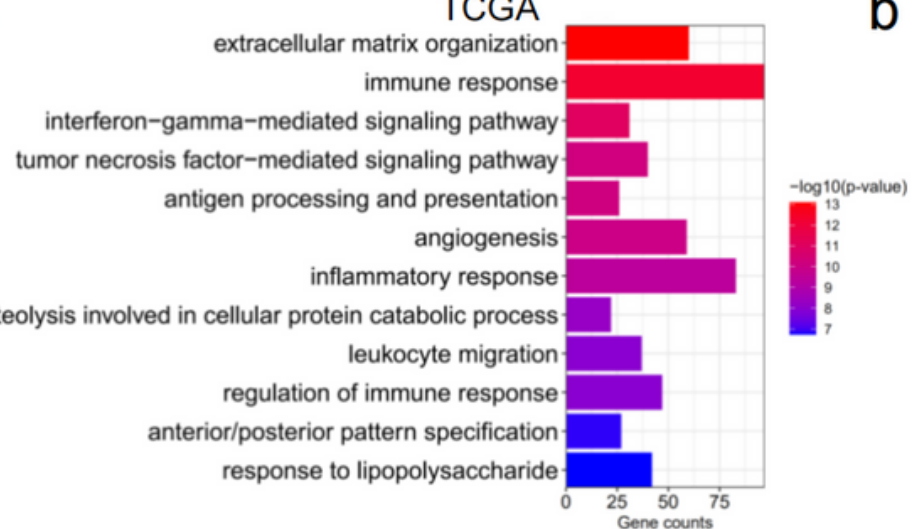

b

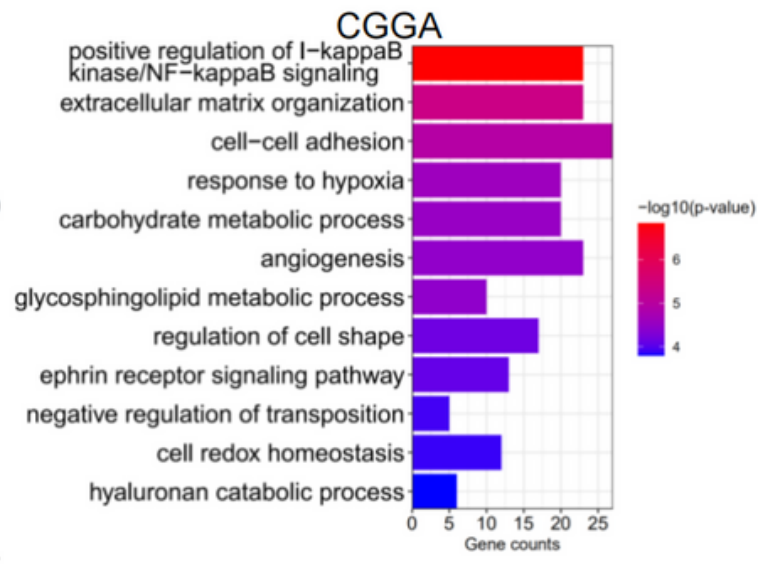

C

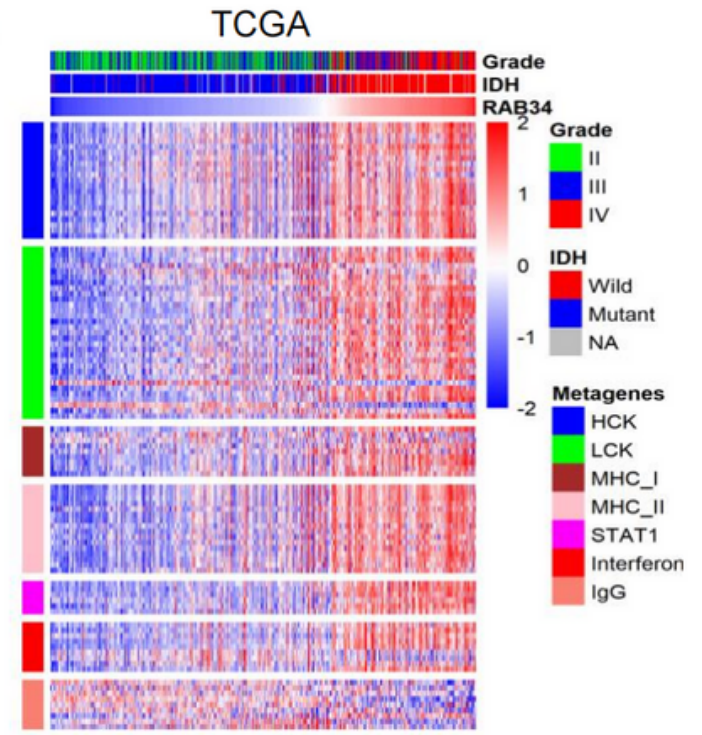

e

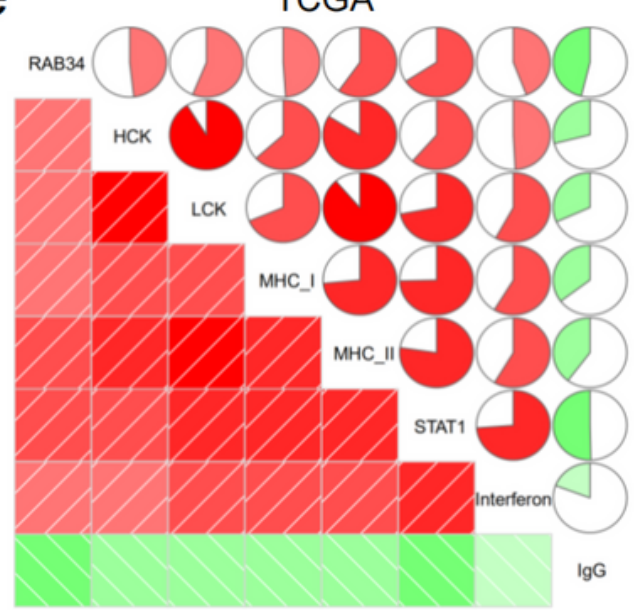

d

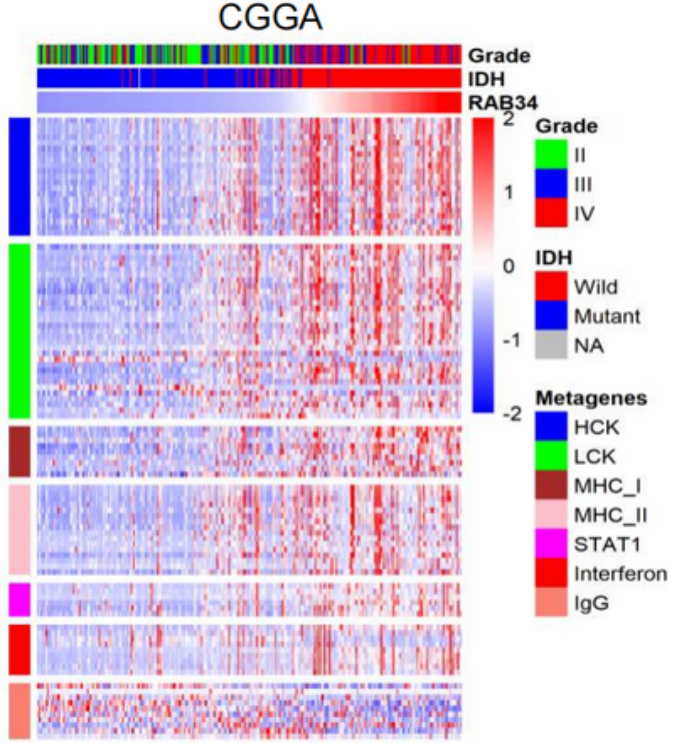

f

CGGA

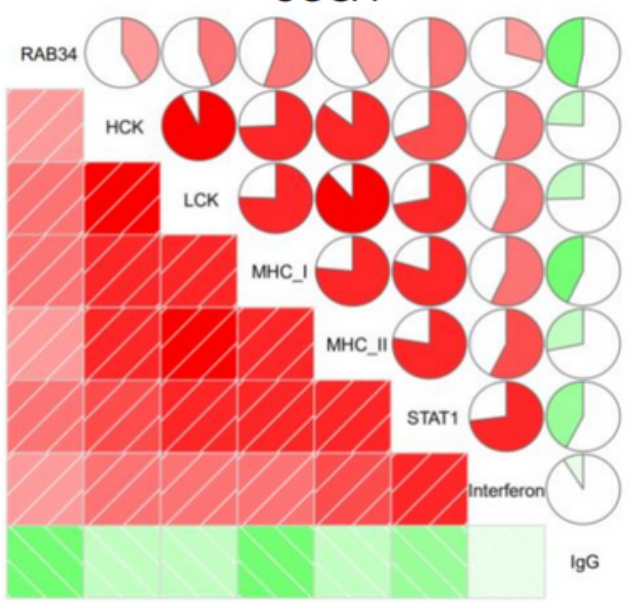

Figure 4

RAB34 expression is related to immune functions and inflammatory activities in glioma. a GO analysis of the TCGA dataset showed that RAB34 was mainly involved in extracellular matrix organization, immune response, angiogenesis, and inflammatory response. b GO analysis of the CGGA dataset showed that 
RAB34 was mainly involved in positive modulation of I-kappaB kinase/NF-kappaB signaling, cell-cell adhesion, extracellular matrix organization, and response to hypoxia. $c$, d The heatmaps of RAB34-related clinicopathological parameters and inflammatory metagenes in TCGA and CGGA cohorts. e, f Corrgrams were established based on the relationship between RAB34 expression and seven inflammatory metagenes.
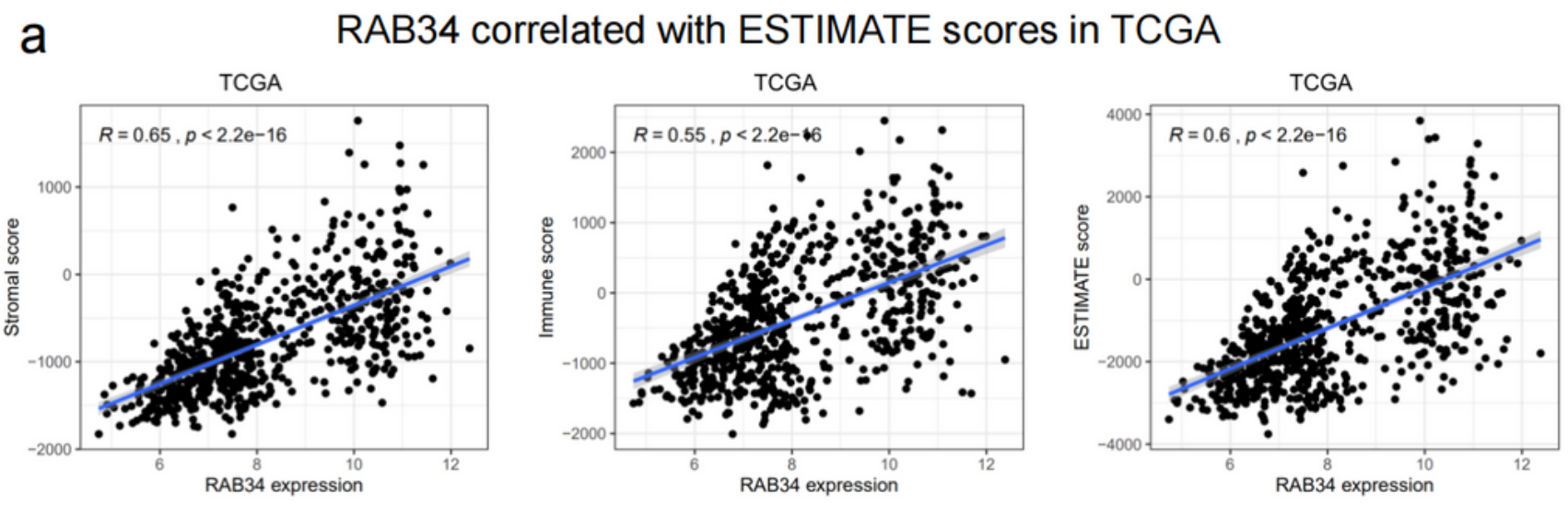

b

RAB34 correlated with ESTIMATE scores in CGGA
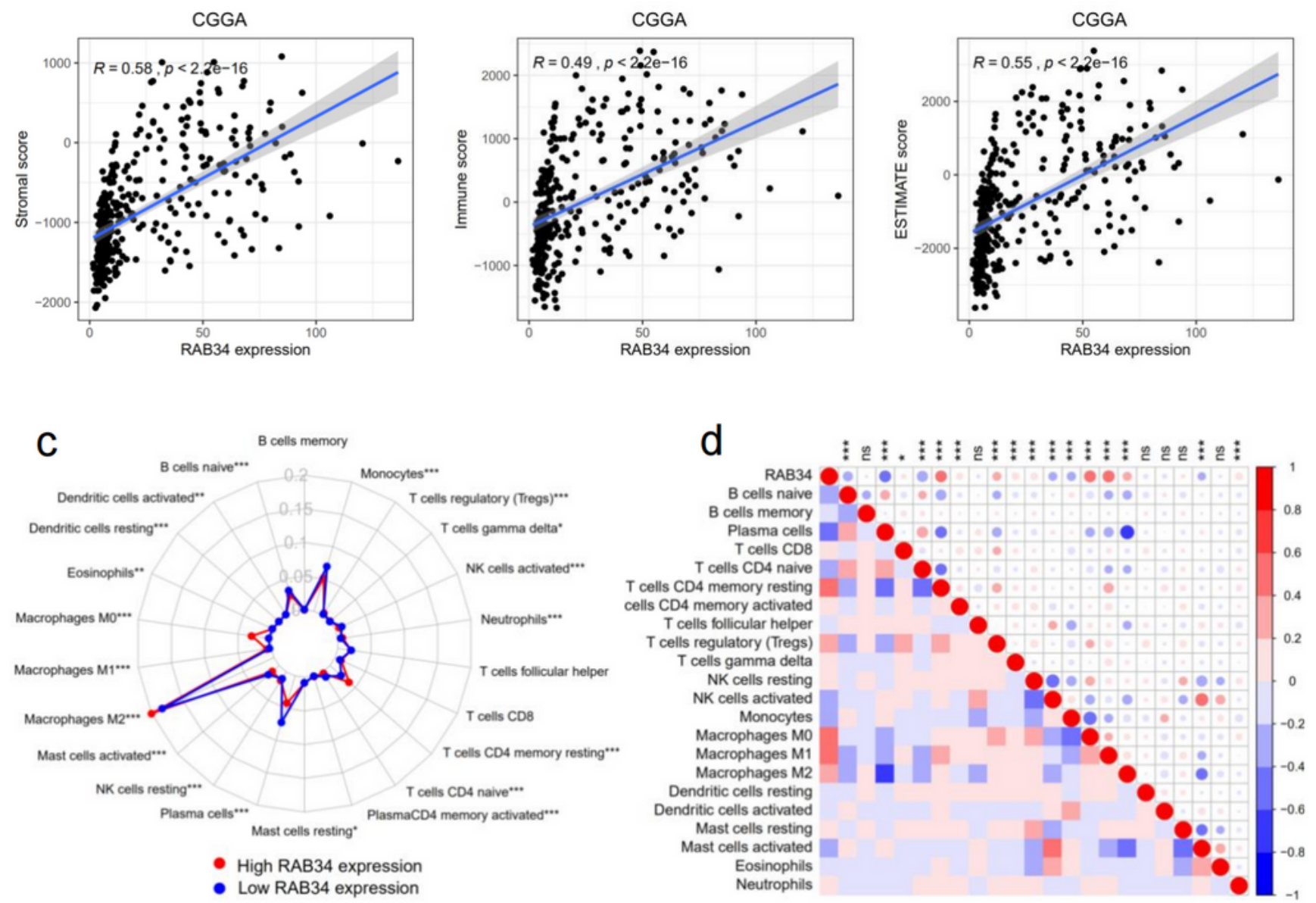

Figure 5 
RAB34 expression is tightly associated with immune infiltration in glioma. a, b RAB34 expression is positively correlated with immune score, stromal score and ESTIMATE score in TCGA and CGGA datasets. c A comparative summary of the percentages of immune cells in different RAB34 expression groups estimated by CIBERSORT in TCGA dataset. d RAB34 expression is significantly correlated with infiltrated cells in the glioma microenvironment. ns, not significant; * $\mathrm{P}<0.05$; $* * \mathrm{P}<0.01$; *** $\mathrm{P}<0.001$.
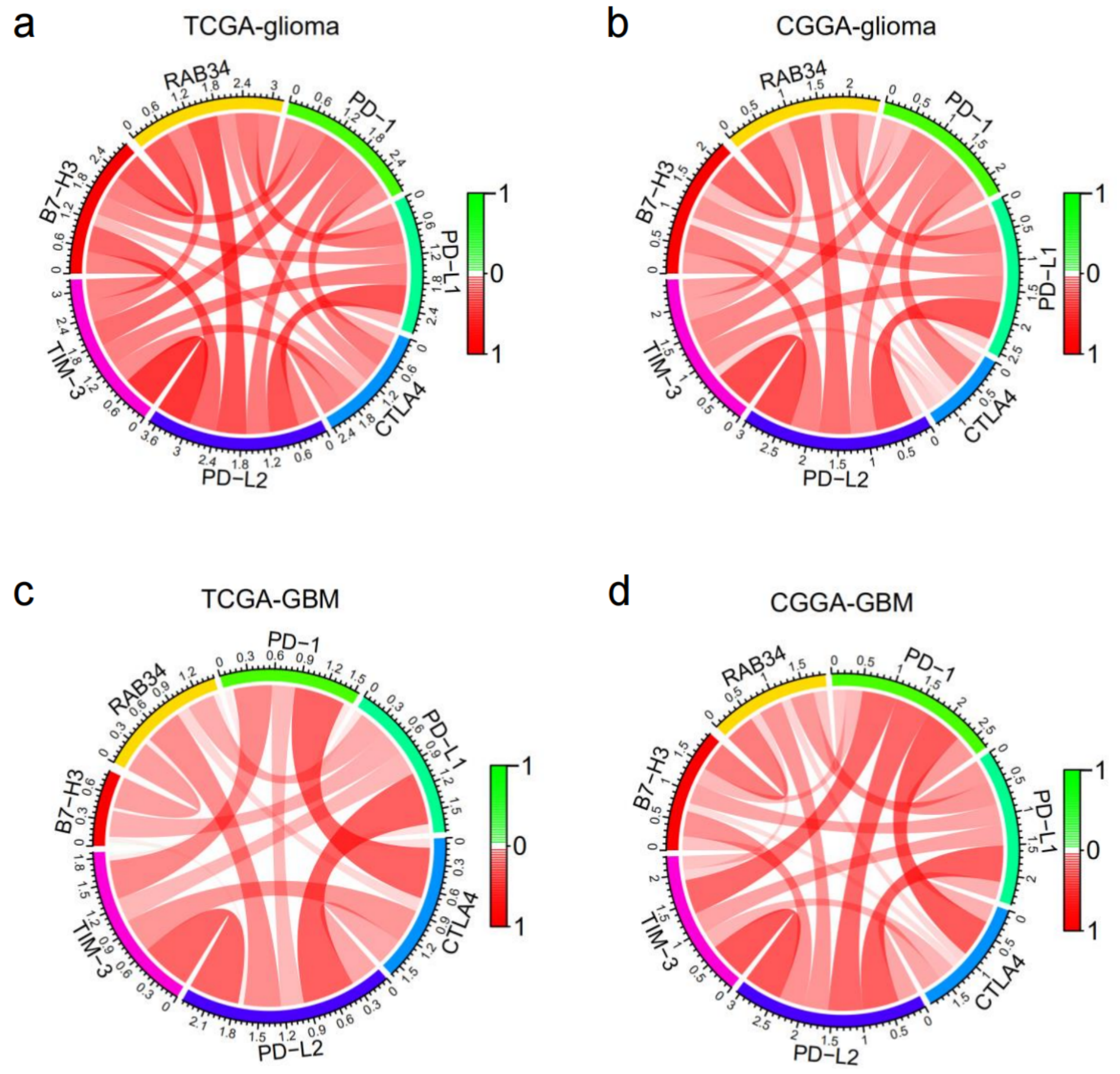

Figure 6

RAB34 expression is correlated with immune checkpoints. a, b Correlation between RAB34 and immune checkpoints in whole-grade gliomas in TCGA and CGGA datasets. c, d Correlation between RAB34 and 
immune checkpoints in GBM in TCGA and CGGA datasets.

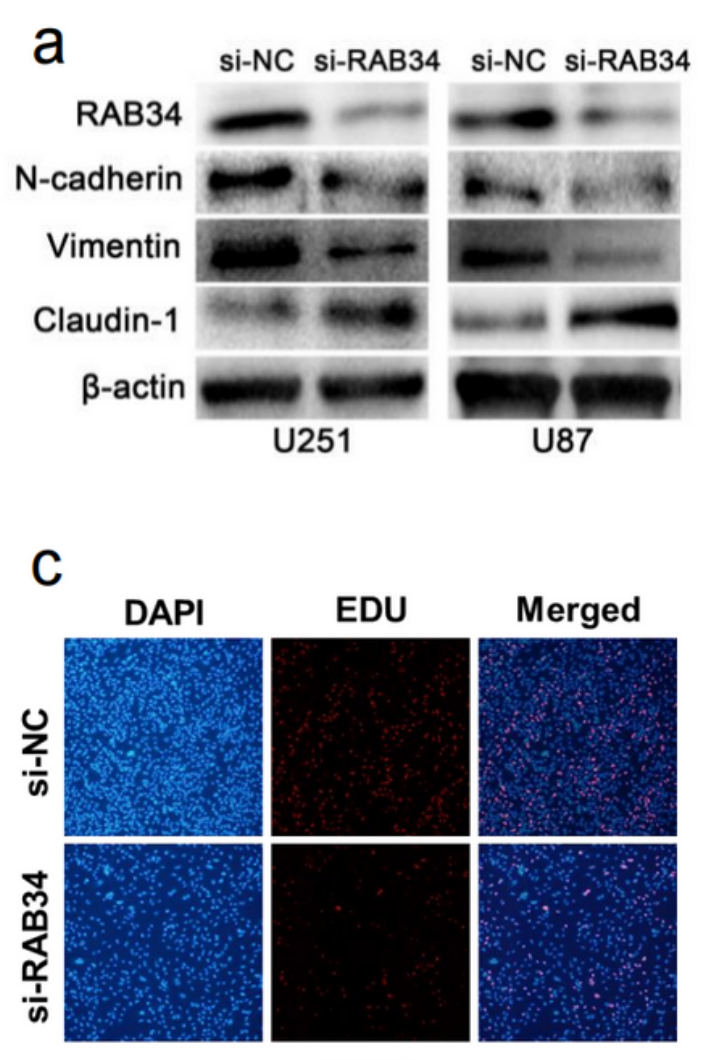

U251 b

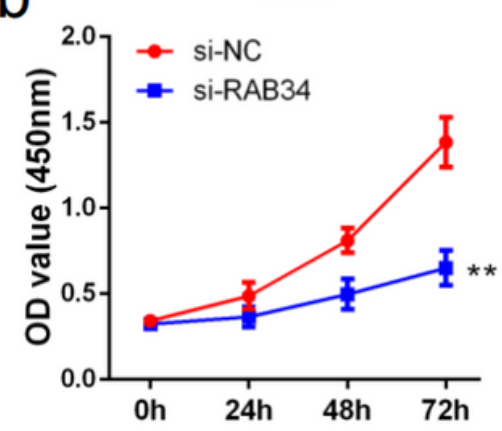

U87

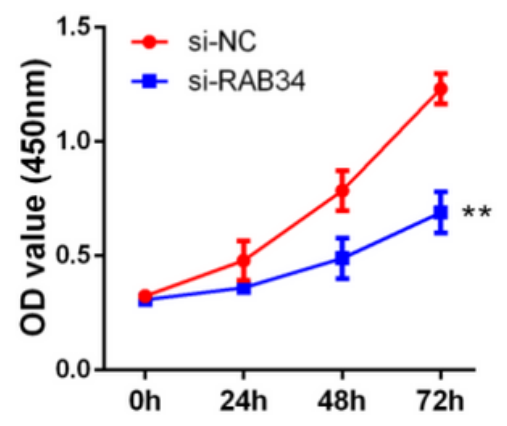

U251

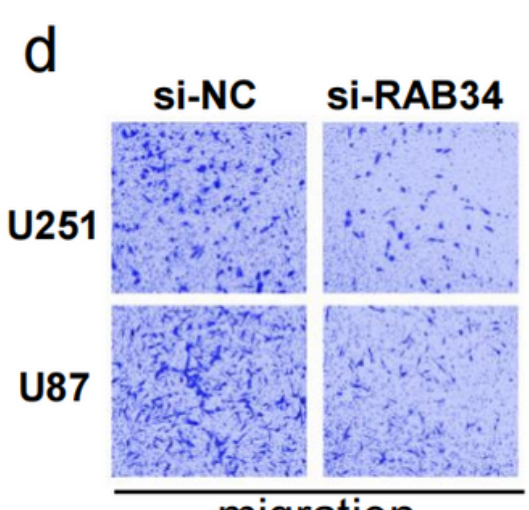

migration
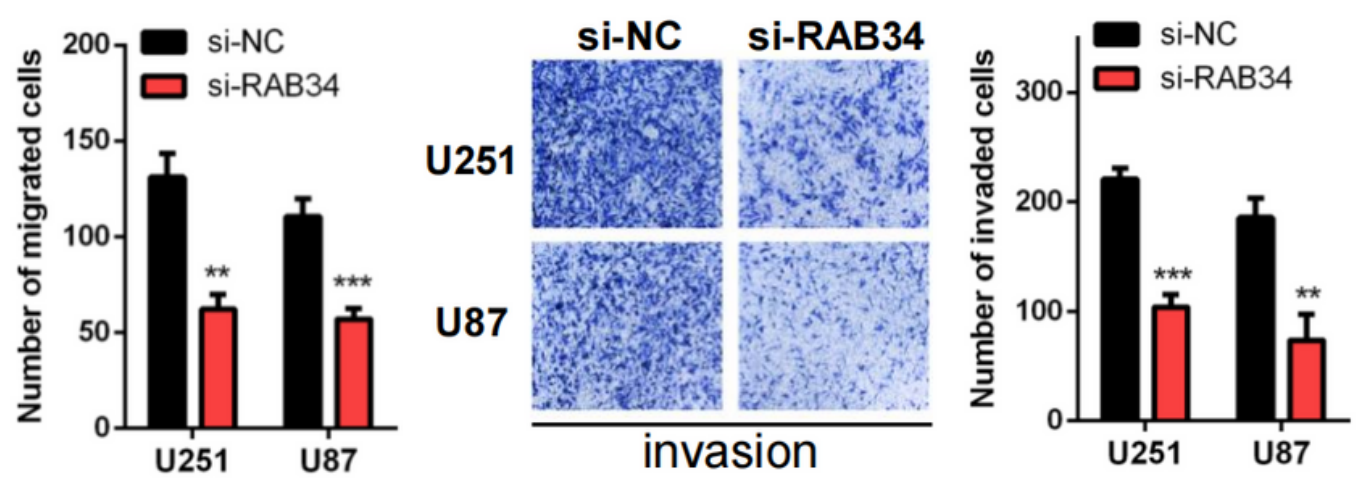

Figure 7

RAB34 promotes the EMT process, proliferation, migration, and invasion of glioma cells. a Western blot assays were performed to detect the protein levels of RAB34, N-cadherin, Vimentin, and Claudin- 1 after RAB34 knockdown in U251 and U87 cells. b CCK-8 assays showed that suppression of RAB34 inhibited cell viability in U251 and U87 cells. c EdU assays showed that suppression of RAB34 inhibited cell proliferation in U251 and U87 cells. d Transwell assays showed that reduction of RAB34 repressed the migration and invasion abilities of U251 and U87 cells, respectively. ** $P<0.01$; *** $P<0.001$. 

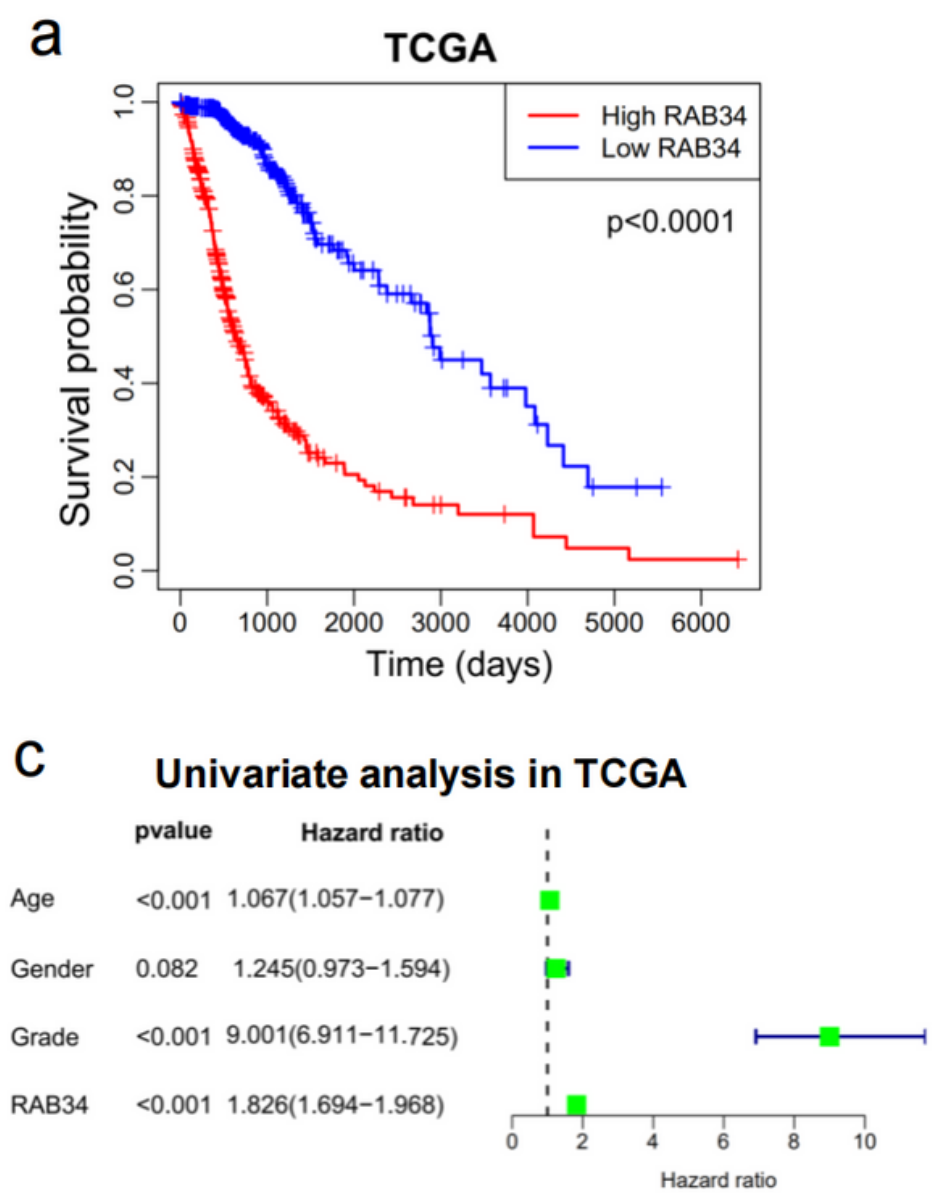

b

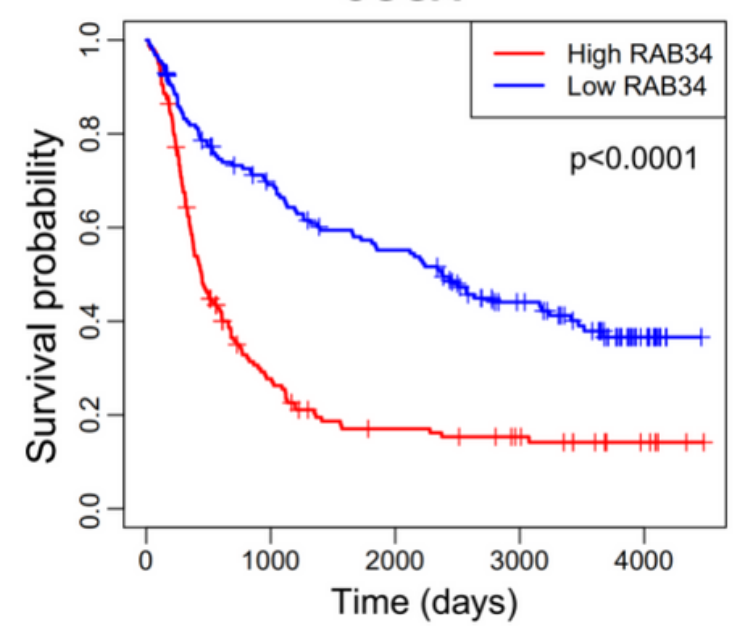

d Univariate analysis in CGGA

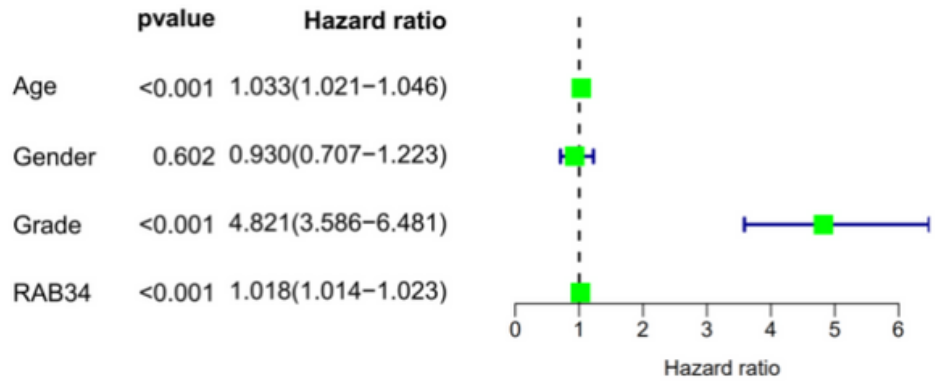

f

\section{Multivariate analysis in TCGA}
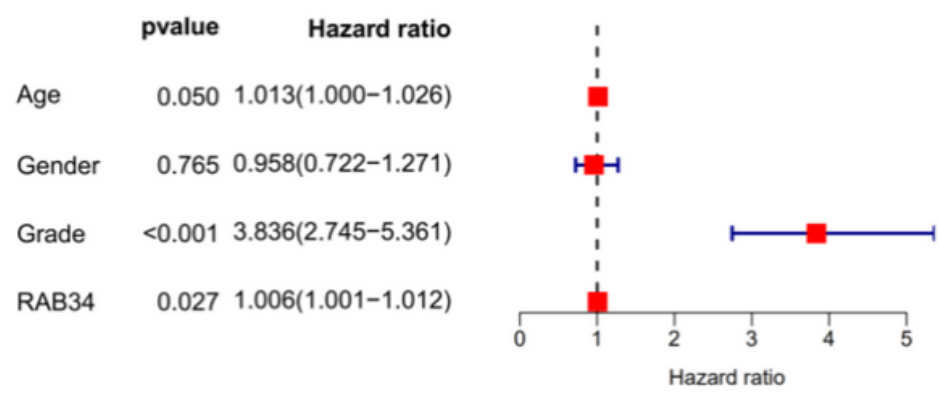

Figure 8

RAB34 predicts worse survival in glioma. a, b Kaplan-Meier survival analyses showed that high RAB34 expression predicted poor prognosis for glioma patients based on the TCGA and CGGA datasets. c, d Univariate Cox regression analyses revealed that RAB34 was associated with overall survival of glioma patients in TCGA and CGGA datasets. e, f Multivariate Cox regression analyses revealed that RAB34 was an independent prognostic biomarker for glioma patients in TCGA and CGGA datasets.

\section{Supplementary Files}

This is a list of supplementary files associated with this preprint. Click to download. 
- FigureS1.png

- Figures2.png

- Supplementaryfigurelegend.docx 